\title{
Correction to: The evolution of the ventilatory ratio is a prognostic factor in mechanically ventilated COVID-19 ARDS patients
}

\author{
Antoni Torres ${ }^{1,2,53^{*}+} \odot$, Anna Motos ${ }^{1,2 \dagger}$, Jordi Riera ${ }^{3}$, Laia Fernández-Barat ${ }^{1,2 \dagger}$, Adrián Ceccato ${ }^{1 \dagger}$, \\ Raquel Pérez-Arnal ${ }^{4}$, Dario García-Gasulla ${ }^{4}$, Oscar Peñuelas ${ }^{1,5}$, José Angel Lorente ${ }^{1,5}$, Alejandro Rodriguez ${ }^{6}$, \\ David de Gonzalo-Calvo ${ }^{1,7}$, Raquel Almansa ${ }^{8,9}$, Albert Gabarrús ${ }^{2}$, Rosario Menéndez ${ }^{10}$, \\ Jesús F. Bermejo-Martin ${ }^{8,9}$, Ricard Ferrer ${ }^{3}$, Rosario Amaya Villar ${ }^{11}$, José M. Añón ${ }^{1,12}$, Carme Barberà ${ }^{13}$, \\ José Barberán ${ }^{14}$, Aaron Blandino Ortiz ${ }^{15}$, Elena Bustamante-Munguira ${ }^{16}$, Jesús Caballero ${ }^{17}$, Cristina Carbajales ${ }^{18}$, \\ Nieves Carbonell ${ }^{19}$, Mercedes Catalán-González ${ }^{20}$, Cristóbal Galbán ${ }^{21}$, Víctor D. Gumucio-Sanguino ${ }^{22,23,}$ \\ Maria del Carmen de la Torre ${ }^{24}$, Emili Díaz ${ }^{25,26}$, Ángel Estella ${ }^{27}$, Elena Gallego ${ }^{28}$, José Luis García Garmendia ${ }^{29}$, \\ José Garnacho-Montero ${ }^{30}$, José M. Gómez ${ }^{31}$, Arturo Huerta ${ }^{32}$, Ruth Noemí Jorge García ${ }^{33}$, Ana Loza-Vázquez ${ }^{34}$, \\ Judith Marin-Corral ${ }^{35}$, Amalia Martínez de la Gándara ${ }^{36}$, Ignacio Martínez Varela ${ }^{37}$, Juan López Messa ${ }^{38}$, \\ Guillermo M. Albaiceta1 ${ }^{1,39,40}$, Mariana Andrea Novo ${ }^{41}$, Yhivian Peñasco ${ }^{42}$, Juan Carlos Pozo-Laderas ${ }^{43}$, \\ Pilar Ricart ${ }^{44}$, Inmaculada Salvador-Adell ${ }^{45}$, Angel Sánchez-Miralles ${ }^{46}$, Susana Sancho Chinesta ${ }^{47}$, \\ Lorenzo Socias ${ }^{48}$, Jordi Solé-Violan ${ }^{49}$, Fernando Suares Sipmann ${ }^{50}$, Luis Tamayo Lomas ${ }^{51}$, José Trenado ${ }^{52}$ and \\ Ferran Barbé ${ }^{1,7}$ on behalf of CIBERESUCICOVID Project (COV20/00110, ISCIII)
}

\section{Correction to: Crit Care (2021) 25:331}

https://doi.org/10.1186/s13054-021-03727-x

Following publication of the original article [1], the authors identified an error in the Funding section.

The updated Funding section is given below and the changes have been highlighted in bold typeface.

The original article can be found online at https://doi.org/10.1186/s13054021-03727-x

\section{*Correspondence: atorres@clinic.cat}

${ }^{\dagger}$ Antoni Torres and Anna Motos have contributed equally to this work and are considered as co-first authors; Laia Fernández-Barat and Adrián

Ceccato have also contributed equally to this work and are considered as co-third authors

${ }^{1}$ Centro de Investigación Biomedica En Red - Enfermedades Respiratorias (CIBERES), Barcelona, Spain

Full list of author information is available at the end of the article

\section{Funding}

Financial support was provided by the Instituto de Salud Carlos III de Madrid (COV20/00110, ISCIII), Fondo Europeo de Desarrollo Regional (FEDER), "Una manera de hacer Europa", and by the Centro de Investigación Biomedica En Red - Enfermedades Respiratorias (CIBERES). DdGC has received financial support from Instituto de Salud Carlos III (Miguel Servet 2020: CP20/00041), co-funded by European Social Fund (ESF)/"Investing in your future".

The original article [1] has been corrected. permits use, sharing, adaptation, distribution and reproduction in any medium or format, as long as you give appropriate credit to the original author(s) and the source, provide a link to the Creative Commons licence, and indicate if changes were made. The images or other third party material in this article are included in the article's Creative Commons licence, unless indicated otherwise in a credit line to the material. If material is not included in the article's Creative Commons licence and your intended use is not permitted by statutory regulation or exceeds the permitted use, you will need to obtain permission directly from the copyright holder. To view a copy of this licence, visit http://creativecommons.org/licenses/by/4.0/. The Creative Commons Public Domain Dedication waiver (http://creativecommons.org/publicdomain/zero/1.0/) applies to the data made available in this article, unless otherwise stated in a credit line to the data. 


\section{Author details}

${ }^{1}$ Centro de Investigación Biomedica En Red - Enfermedades Respiratorias (CIBERES), Barcelona, Spain. ${ }^{2}$ Institut d'Investigacions August Pi i Sunyer (IDIBAPS), Universitat de Barcelona, Barcelona, Spain. ${ }^{3}$ Intensive Care Department, Hospital Universitari Vall d'Hebron, Vall d'Hebron Institut de Recerca, Barcelona, Spain. ${ }^{4}$ Barcelona Supercomputing Center (BSC), Barcelona, Spain. ${ }^{5}$ Hospital Universitario de Getafe, Universidad Europea, Madrid, Spain. ${ }^{6}$ Critical Care Department, Hospital Joan XXIII, Tarragona, Spain. ${ }^{7}$ Translational Research in Respiratory Medicine, Respiratory Department, Hospital Universitari Aranu de Vilanova and Santa Maria, IRBLleida, Lleida, Spain. ${ }^{8}$ Hospital Universitario Río Hortega de Valladolid, Valladolid, Spain. ${ }^{9}$ Instituto de Investigación Biomédica de Salamanca (IBSAL), Gerencia Regional de Salud de Castilla y León, Salamanca, Spain. ${ }^{10}$ Pulmonary Department, University and Polytechnic Hospital La Fe, Valencia, Spain. ${ }^{11}$ Intensive Care Clinical Unit, Hospital Universitario Virgen de Rocío, Sevilla, Spain. ${ }^{12}$ Servicio de Medicina Intensiva, Hospital Universitario La Paz, IdiPAZ, Madrid, Spain. ${ }^{13}$ Hospital Santa Maria, IRBLleida, Lleida, Spain. ${ }^{14}$ Hospital Universitario HM Montepríncipe, Universidad San Pablo-CEU, Madrid, Spain. ${ }^{15}$ Servicio de Medicina Intensiva, Hospital Universitario Ramón y Cajal, Madrid, Spain. ${ }^{16}$ Department of Intensive Care Medicine, Hospital Clínico Universitario Valladolid, Valladolid, Spain. ${ }^{17}$ Critical Care Department, Hospital Universitari Arnau de Vilanova, IRBLleida, Lleida, Spain. ${ }^{18}$ Hospital Álvaro Cunqueiro, Vigo, Spain. ${ }^{19}$ Intensive Care Unit, Hospital Clínico y Universitario de Valencia, Valencia, Spain. ${ }^{20}$ Department of Intensive Care Medicine, Hospital Universitario, 12 de Octubre, Madrid, Spain. ${ }^{21}$ Department of Medicine, CHUS, Complejo Hospitalario Universitario de Santiago, Santiago de Compostela, Spain. ${ }^{22}$ Department of Intensive Care, Hospital Universitari de Bellvitge, L'Hospitalet de Llobregat, Barcelona, Spain. ${ }^{23}$ Bellvitge Biomedical Research Institute (IDIBELL), L'Hospitalet de Llobregat, Barcelona, Spain. ${ }^{24}$ Hospital de Mataró de Barcelona, Barcelona, Spain. ${ }^{25}$ Department of Medicine, Universitat Autònoma de Barcelona (UAB), Barcelona, Spain. ${ }^{26} \mathrm{Critical}$ Care Department, Corporació Sanitària Parc Taulí, Sabadell, Barcelona, Spain. ${ }^{27}$ Departamento Medicina Facultad Medicina, Universidad de Cádiz, Hospital Universitario de Jerez, Jerez de la Frontera, Spain. ${ }^{28}$ Unidad de Cuidados Intensivos, Hospital San Pedro de Alcántara, Cáceres, Spain. ${ }^{29}$ Intensive Care Unit, Hospital San Juan de Dios del Aljarafe, Sevilla, Spain. ${ }^{30}$ Intensive Care Clinical Unit, Hospital Universitario Virgen Macarena, Seville, Spain. ${ }^{31}$ Hospital General Universitario Gregorio Marañón, Madrid, Spain. ${ }^{32}$ Pulmonary and Critical Care Division, Emergency Department, Clínica Sagrada Família, Barcelona, Spain.

${ }^{33}$ Intensive Care Department, Hospital Nuestra Señora de Gracia, Zaragoza,
Spain. ${ }^{34}$ Unidad de Medicina Intensiva, Hospital Universitario Virgen de Valme, Sevilla, Spain. ${ }^{35}$ Critical Care Department, Hospital del Mar-IMIM, Barcelona, Spain. ${ }^{36}$ Department of Intensive Medicine, Hospital Universitario Infanta Leonor, Madrid, Spain. ${ }^{37}$ Critical Care Department, Hospital Universitario Lucus Augusti, Lugo, Spain. ${ }^{38}$ Complejo Asistencial Universitario de Palencia, Palencia, Spain. ${ }^{39}$ Departamento de Biología Funcional, Instituto Universitario de Oncología del Principado de Asturias, Universidad de Oviedo, Oviedo, Spain. ${ }^{40}$ Instituto de Investigación Sanitaria del Principado de Asturias, Hospital Central de Asturias, Oviedo, Spain. ${ }^{41}$ Servei de Medicina Intensiva, Hospital Universitari Son Espases, Palma de Mallorca, Illes Balears, Spain. ${ }^{42}$ Servicio de Medicina Intensiva, Hospital Universitario Marqués de Valdecilla, Santander, Spain. ${ }^{43}$ UGC-Medicina Intensiva, Hospital Universitario Reina Sofia, Instituto Maimonides IMIBIC, Córdoba, Spain. ${ }^{44}$ Servei de medicina intensiva, Hospital Universitari Germans Trias, Badalona, Spain. ${ }^{45}$ Hospital Verge de La Cinta, Tortosa, Tarragona, Spain. ${ }^{46}$ Hospital de Sant Joan d'Alacant, Alacant, Spain. ${ }^{47}$ Servicio de medicina intensiva, Hospital Universitario y Politécnico La Fe, Valencia, Spain. ${ }^{48}$ Intensive Care Unit, Hospital Son Llàtzer, Palma de Mallorca, Illes Balears, Spain. ${ }^{49}$ Critical Care Department, Hospital Dr. Negrín Gran Canaria, Las Palmas, Gran Canaria, Spain. ${ }^{50}$ Intensive Care Unit, Hospital Universitario La Princesa, Madrid, Spain. ${ }^{51}$ Critical Care Department, Hospital Universitario Río Hortega de Valladolid, Valladolid, Spain. ${ }^{52}$ Servicio de Medicina Intensiva, Hospital Universitario Mútua de Terrassa, Terrassa, Barcelona, Spain. ${ }^{53}$ Servei de Pneumologia i Al.lèrgia Respiratòria, Hospital Clínic, Villarroel 170, Esc 6/8 Planta 2, 08036 Barcelona, Spain.

\section{Published online: 17 December 2021}

\section{Reference}

1. Torres A, Motos A, Riera J, et al. The evolution of the ventilatory ratio is a prognostic factor in mechanically ventilated COVID-19 ARDS patients. Crit Care. 2021;25:331. https://doi.org/10.1186/s13054-021-03727-x.

\section{Publisher's Note}

Springer Nature remains neutral with regard to jurisdictional claims in published maps and institutional affiliations. 